\title{
MicroRNA-492 overexpression exerts suppressive effects on the progression of osteosarcoma by targeting PAK7
}

\author{
XUANHE SONG $^{1}$, YAOPING XIE ${ }^{2}$, YANG LIU $^{1}$, MING SHAO $^{1}$ and WEILIANG YANG ${ }^{1}$ \\ Departments of ${ }^{1}$ Orthopaedic Surgery and ${ }^{2}$ Nuclear Medicine, The First Affiliated Hospital of \\ Harbin Medical University, Harbin, Heilongjiang 150001, P.R. China
}

Received January 18, 2016; Accepted May 24, 2017

DOI: $10.3892 /$ ijmm.2017.3046

\begin{abstract}
MicroRNAs (miRNAs or miRs), which are a class of non-coding RNAs, have emerged as effective modulators of various aspects of biological processes. Accumulating evidence has established significant associations between the dysregulation of miRs and tumorigenesis in various types of cancer. However, the role of miR-492, particularly in osteosarcoma (OS) remains elusive. In present study, we demonstrated that miR-492 functions as putative tumor suppressor miR in OS. The level of miR-492 was frequently downregulated in both OS tissues and cell lines. Moreover, the ectopic overexpression of miR-492 effectively inhibited the proliferation, migration and invasion of OS cell lines. Furthermore, transfection with a miR-492 overexpression vector also strongly attenuated the growth of xenograft tumors in vivo. p21-activated kinase (PAK7) was identified as the putative target of miR-492 in OS, and we further found a significantly inverse correlation between PAK7 and miR-492 in OS specimens. Taken together, our study has unraveled a novel role for miR-492 in OS and may help in establishing the rationale for more effective treatment strategies for OS via miR regulation.
\end{abstract}

\section{Introduction}

Tumorigenesis has been a serious threat to human health and survival for decades and among the malignant cancers, osteosarcoma (OS) is one of the most common tumors, particularly in adolescents (1). The occurrence of OS denotes a complex mechanism where multiple factors from environmental and genetic origins may largely contribute to the complexity (2). During the development of OS, immature bones are formed and normal bones are jeopardized (3). In addition, long bones are at a greater risk of the onset of OS, particularly those in

Correspondence to: Dr Weiliang Yang, Department of Orthopaedic Surgery, The First Affiliated Hospital of Harbin Medical University, 23 Youzheng Street, Nangang, Harbin, Heilongjiang 150001, P.R. China

E-mail: yangwl_hmu@163.com

Key words: microRNA-492, p21-activated kinase 7, osteosarcoma, migration, invasion the knees (4). The survival rates of patients with OS are particularly low despite significant improvements being made, including combinatorial chemotherapy and surgery $(5,6)$. The mechanisms responsible for the development of OS are very complex. Therefore, the in-depth understanding of the occurrence and development of OS poses a new challenge to biological researchers.

The p21-activated kinases (PAKs) belong to the serine/threonine kinases which can be activated by GTPases (7). PAKs are classified into 2 subgroups, namely group I PAKs (PAK1-3) and group II PAKs (PAK4-6), while PAK7 is a different type of PAK compared with group I and group II (8). PAKs are implicated in massive biological processes, particularly in neoplasms (7). The expression of PAK7 is usually found to be upregulated in diverse types of cancer, including OS, lung cancer, colorectal cancer and pancreatic cancer (9-13). Therefore, the oncogene $P A K 7$ may be implicated in the progression of numerous types of cancer.

A class of non-coding RNAs with a short length known as microRNAs (miRNAs of miRs) (approximately 22 nucleotides in length) are usually evolutionarily conserved and play pivotal roles in various biological processes (14). Complementary binding to the 3'-untranslated region (3'-UTR) of target mRNAs can either silence the translation or promote their degradation (15). The involvement of miRs has been reported in numerous types of cancer, such as lung cancer, hepatocellular carcinoma (HCC), breast cancer and colon cancer (16-21). The role of miRs in tumorigenesis is complex, partly due to the fact that miRs can either function as tumor suppressors or oncogenes (17-19,22-24). For example, He et al suggested that miR-34 inhibits the development of OS in a p53-dependent manner (25). However, Li et al demonstrated that miR-296-5p induced the proliferation of gastric cancer cells by targeting caudal-related homeobox 1 (CRH1) (26). The expression and epigenetics of miRs are usually altered in tumorous tissues $(19,27)$. These findings suggest that miRs are intensively implicated in tumorigenesis. However, even though numerous studies have been carried out on miRs and cancer, the association between miR-492 and OS remains poorly understood.

In this study, we found that miR-492 was usually downregulated in both OS cell lines and human specimens. The ectopic expression of miR-492 significantly attenuated the adverse effects on OS, inhibiting the proliferation, migration and invasion of U-2 OS and MG-63 cells in vitro. Furthermore, 
transfection with a miR-492 overexpression vector also decreased the tumor volume in an implantation assay in vivo. We further identified PAK7 as a potential miR-492 target in OS. The inverse correlation between PAK7 and miR-492 was also evident in tumorous tissues. Given the oncogenic role of PAK7, to the best of our knowedge, we are the first to demonstrate that miR-492 can serve as a candidate tumor suppressor by targeting PAK7 in OS. Our findings provided important insight into the miR-targeted therapy of OS.

\section{Materials and methods}

Cell culture and human specimens. The OS cell lines, Saos-2, HOS, 143B,U-2 OS, MG-63 and G292, as well as the control osteoblast cell line, hFOB, were purchased from the American Type Culture Collection (ATCC; Rockville, MD, USA). The ZK-58 cells were obtained from Shanghai Institute of Biochemistry and Cell Biology (Shanghai, China). The cells were cultured in RPMI-1640 medium obtained from Sigma-Aldrich (Shanghai, China). The medium was further supplemented with $5 \%$ fetal bovine serum (FBS) and penicillin $(100 \mathrm{U} / \mathrm{ml})$ (Sigma-Aldrich) in a humidified atmosphere of $5 \% \mathrm{CO}_{2}$ at $37^{\circ} \mathrm{C}$. The OS specimens were all surgical archives from patients (age, 41-79; $n=77$ ) at the First Affiliated Hospital of Harbin Medical University (Harbin, China) obtained between September 2013 and July 2015. Paired normal adjacent tissues were resected as controls. All specimens were kept in liquid nitrogen at $-80^{\circ} \mathrm{C}$ following resection. All patients provided written informed consent for their use of their samples. The protocols of the experimental procedures related to human samples were formally approved by the Human Research Ethics Committee of the First Affiliated Hospital of Harbin Medical University (no. 2013F0012).

Reverse transcription-quantitative PCR (RT-qPCR). Total RNA was extracted from both cell lines and human specimens using TRIzol reagent(Invitrogen Life Technologies, Carlsbad, CA, USA) according to the manufacturer's instructions. The cDNA ( $20 \mu \mathrm{g}$ in total) obtained by reverse transcription was generated by the SYBR Premix Taq ${ }^{\text {TM }}$ Toolkit (Takara Bio, Inc., Otsu, Japan) according to the manufacturer's instructions. The TaqMan microRNA qRT-PCR kit (Applied Biosystems, Foster City, CA, USA) was used in present study. To measure $P A K 7$ mRNA expression, the SYBR-Green PCR Master kit was utilized (Takara Bio, Inc.). GAPDH was used as the control. Reactions were performed with the ABI PRISM ${ }^{\circledR} 7000$ Sequence Detection system (Applied Biosystems) following the manufacturer's instructions. miR-492 and PAK7 expression levels were determined using the $2^{-\Delta \Delta \mathrm{Ct}}$ method. All experiments were performed in 3 replicates. The primer sequences used were as follows: miR-492, 5'-GGCTATGCTTGAGTACG-3' (forward) and 5'-CTGAGTTAGCGTACGAGT-3' (reverse); GAPDH, 5'-CTCGCCGCAGTGCATTCGT-3' (forward) and 5'-ACGCTTCGCGATCGTGCGTGAT-3' (reverse); and $P A K 7$, 5'-GCTACGTAGACCCTGAT-3' (forward) and 5'-CAGT CACTCCGTACGG-3' (reverse).

Generation of stably transfected cell lines. The lentiviral system to ectopically overexpress miR-492 in U-2 OS and MG-63 was used in this study. The lentivirus with miR-492 mimics (miR-492) and negative controls were synthesized and purchased from
Sigma-Aldrich. The Lipofectamine ${ }^{\mathrm{TM}} 2000$ system (Invitrogen Life Technologies, Shanghai, China) was used for viral transfection. At $24 \mathrm{~h}$ post-transfection, the cultured medium was replaced with fresh medium. All plasmids were experimentally verified by RT-qPCR.

Luciferase reporter assay. The 3'-UTR region for PAK7 was amplified by PCR using the primer sequences as follows: 5'-ATGCCGTCGCCTCTTGTGTCTTC-3' (forward) and 5'-GTACTGAGTCCTTCTGAGGC-3 (reverse). GAPDH was used as a control. The 3'-UTR for $P A K 7$ with predicted binding sites for hsa-miR-492 was cloned into the Xbal downstream of Renilla luciferase reporter plasmid phRL-TK (Promega, Madison, WI, USA) to obtain the wild-type $P A K 7$ luciferase plasmids (PAK7 3'-UTR WT). A construction with $P A K 7$ point mutation in 3'-UTR was similarly obtained (PAK73'-UTRMUT). 293 T cells (Shanghai Institute of Biochemistry and Cell Biology) were loaded into a 96 -well plate $24 \mathrm{~h}$ prior to transfection and then co-transfected with PAK7 3'-UTR WT or MUT vector. The $\beta$-gal control plasmid (Ambion, Carlsbad, CA, USA), miR-492 or control vectors were transfected into the cells using the Lipofectamine 2000 system (Invitrogen Life Technologies). The Luciferase activities were measured using the Dual-Luciferase reporter system (Promega) as relative luciferase units following the manufacturer's instructions.

Colony formation assay. At 2 days following transfection, at total of $400 \mathrm{U}-2$ OS cells were seeded into a 12-well plate. The plate was replenished with fresh medium at an interval of 2 days. After 2 weeks, the clones were washed with phosphate-buffered saline (PBS) and fixed in 5\% paraformaldehyde (PFA) for $15 \mathrm{~min}$ at $37^{\circ} \mathrm{C}$. Giemsa staining (no. G9641; Sigma-Aldrich) was then performed for $20 \mathrm{~min}$ and the cells were washed with water.

Invasion assay. The upper chamber of a Transwell (8-mm pore size; EMD Millipore, Billerica, MA, USA) was pre-coated with Matrigel (Invitrogen Life Technologies) overnight. After $24 \mathrm{~h}$, the U-2 OS and MG-63 cells transfected with lentivirus were suspended and loaded in the upper chamber $\left(10^{4}\right.$ cells/well $)$ in RPMI-1640 medium (Sigma-Aldrich). The lower chambers were supplemented with RPMI-1640 medium with additional $5 \%$ FBS. After a further $24 \mathrm{~h}$, the upper chamber was removed. The migrating cells into the lower chamber were fixed with 5\% PFA and stained with crystal violet (Sigma-Aldrich). Cells from 5 non-overlapping views were quantified using a Leica microscope fluorescence microscope (DM IRB; Leica Microsystems GmbH, Wetzlar, Germany).

Migration assay. The Boyden chambers (BD Biosciences, San Jose, CA, USA) were used in a 12-well plate. At $24 \mathrm{~h}$ following transfection, $10^{5}$ cells from the RPMI-1640 medium were seeded in the upper chamber (Sigma-Aldrich). RPMI-1640 with $15 \%$ FBS was used as an attractant. Non-migrated cells were removed $24 \mathrm{~h}$ after the experiment, loaded into 5\% PFA and stained with crystal violet.

Proliferation assay. Alterations in cell proliferation were determined using the Cell Counting Kit-8 (CCK-8; Dojindo Molecular Technologies, Inc., Shanghai, China). The U-2 OS 
and MG-63 cells ( $10^{4}$ cell/well) transfected with either lentivirus control or miR-492 lentivirus were seeded on a 96-well plate for 5 days. A total of $20 \mu \mathrm{l}$ MTT solution were added into the culture $(10 \mathrm{mg} / \mathrm{ml})$ for $4 \mathrm{~h}$ at an interval of 1 day. The optical density (OD) at $490 \mathrm{~nm}$ was determined using Spectramax M5 microplate monitor (Molecular Devices, LLC, Sunnyvale, CA, USA) following the manufacturer's instructions.

Cancer cell implantation. In total, $10^{5} \mathrm{U}-2 \mathrm{OS}$ cells transfected with lentiviral miR-492 or lentiviral controls were injected subcutaneously into BALB/c nude mice. In total, 12 mice (age, 4-5 weeks; average weight, $16.6 \mathrm{~g}$; male: 6 , female: 6 ) were used in the current study. Mice were housed at $20^{\circ} \mathrm{C}$, with $50-60 \%$ humidity and a light-dark cycle of $12 \mathrm{~h}$. Ad libitum access to food and water was provided. Animal experiments were formally reviewed and approved by the Ethics Committee for Animal Research at the First Affiliated Hospital of Harbin Medical University. The tumor volume was measured at an interval of 3 days for 30 days. By the end of the implantation, all mice were sacrificed and Ki-67 immunostaining was performed using the KI-67 kit (Sigma-Aldrich) according to the manufacturer's instructions.

Western blot analysis. The U-2 OS and MG-63 cells were harvested with lysis buffer (12\% glycerol and 5\% NP-40) obtained from Sigma-Aldrich. The protein extracts (equally $100 \mu \mathrm{g}$ for each) were dissolved in 10\% SDS-PAGE and transferred onto nitrocellulose membranes (Bio-Rad Laboratories, Hercules, CA, USA). The membranes were coated with monoclonal anti-PAK7 antibody (cat. no. K3265) and antiGAPDH antibody (cat. no. G8795) (both from Sigma-Aldrich) overnight. The HRP-conjugated secondary antibodies $(1: 1,000)$ were supplemented at $20^{\circ} \mathrm{C}$ for $2 \mathrm{~h}$. The chemiluminescence film system (Amersham Pharmacia Biotech, Shanghai, China) was used to visualize the immunoblots.

Predicting miR-492 target. We used algorithms for target gene prediction TargetScan (http://genes.mit.edu/targetscan), DIANA-microT (http://diana.imis.athena-innovation.gr/ DianaTools/index.php?r=microT_CDS/index) and miRDB (www.mirdb.org) as previously described (28-30). Briefly, putative targets were ranked by $\mathrm{Z}$ scores. The top ranked overlapping targets are selected for experimental verification.

Statistical analysis. Statistical results were all analyzed using SPSS software (version 16.0; SPSS, Inc., Chicago, IL, USA). All experiments were carried out in triplicate. P-values $<0.05$ were considered to indicate statistically significant differences. A paired test was used for pair-wise sample comparisons.

\section{Results}

Expression of miR-492 is reduced in OS samples and cell lines. To determine whether the expression of miR-492 is altered in OS, RT-qPCR was performed. The results revealed that the level of miR-492 was significantly downregulated in the OS samples compared with the corresponding paired normal adjacent tissues ( $\mathrm{n}=77, \mathrm{P}<0.01$; Fig. $1 \mathrm{~A})$. In addition, we also investigated the expression of miR-492 in well-established OS cell lines. The results also confirmed that miR-492 was markedly decreased in OS cell lines in contrast to the normal
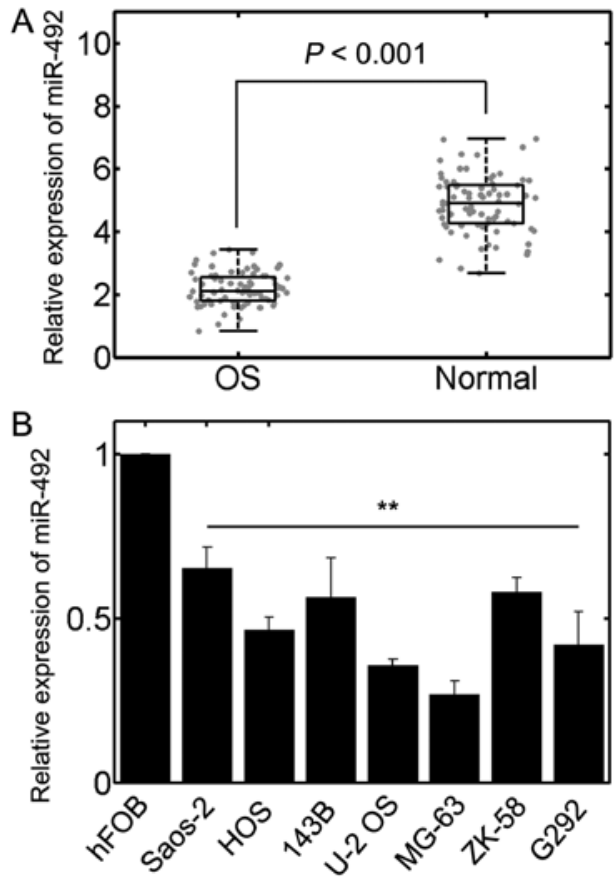

Figure 1. Relative expression of miR-492 in osteosarcoma (OS) specimens and cell lines. (A) Expression of miR-492 in 77 OS specimens compared with normal adjacent tissues $(\mathrm{P}<0.001)$. (B) Expression of miR-492 in normal osteoblast cell lines and OS cell lines. The expression of miR-492 was normalized by endogenous $G A P D H .{ }^{* *} \mathrm{P}<0.01$.

osteoblast cell line, hFOB (Fig. 1B). These results implied that miR-492 is downregulated in OS and may thus play a role in the development of OS. Since the U-2 OS and MG-63 cells exhibited the lowest miR-492 expression, we selected these 2 cell lines for further analysis.

miR-492 inhibits the malignancy of OS in vitro. We then performed experiments to access the role of miR-492 in the proliferation, migration and invasion of OS cells. The U-2 OS and MG-63 cells were transfected with either a control vector or recombinant plasmid encoding miR-492 precursor. The results of RT-qPCR revealed that miR-492 expression was significantly elevated owing to miR-492 precursor transfection (Fig. 2A). In addition, we found that transfection with the miR-492 overexpression vector substantially inhibited the proliferation of both U-2 OS and MG-63 cells (Fig. 2B). The difference in proliferation was evident as early as 3 days in miR-492-overexpressing cells (Fig. 2B). Furthermore, miR-492 also attenuated the migration of both OS cell lines, as accessed by migration assays (Fig. 2C). The efficacy of inhibition was even $>50 \%$ (Fig. $2 \mathrm{C}$, bottom panels). Invasion assays also yielded similar results, which suggested that miR-492 can significantly decrease the invasive capacities of U-2 OS and MG-63 cell lines (Fig. 2D). These results collectively demonstrated that miR-492 can potently inhibit the tumorigenic potential of OS cell lines.

miR-492 modulates the growth of OS tumors. We further used colony formation assays to access the tumorigenic ability of OS cells. We found that the average number of colonies in U-2 OS cells transfected with miR-492 precursor plasmids was significantly decreased $(\mathrm{P}<0.01$; Fig. $3 \mathrm{~A})$. To investigate the role of 

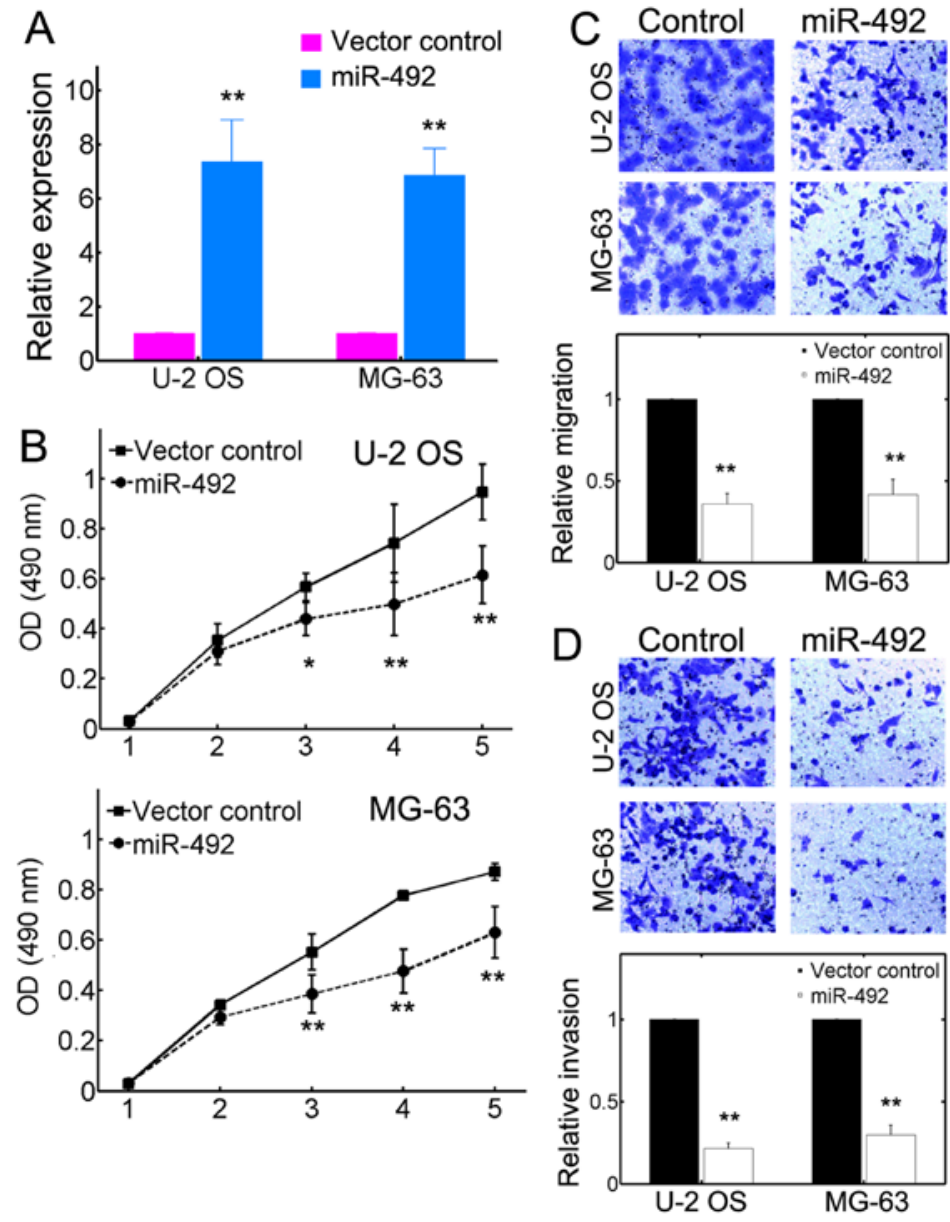

Figure 2. Inhibitory effect of miR-492 in osteosarcoma (OS). (A) The miR-492 level quantified by qPCR in U-2 OS and MG-63 cells transfected with empty control or miR-492 vector. (B) Proliferation of U-2 OS and MG-63 cells transfected with either control vector or miR-492 expression vector. (C) Migration assays for U-2 OS and MG-63 cells transfected with either control vector or miR-492 vector (top panel). Quantification results are shown below. ${ }^{*} \mathrm{P}<0.05,{ }^{* *} \mathrm{P}<0.01$. (D) Representative images of the invasion of U-2 OS and MG-63 cells accessed by Transwell assays. ${ }^{*} \mathrm{P}<0.05,{ }^{* *} \mathrm{P}<0.01$.
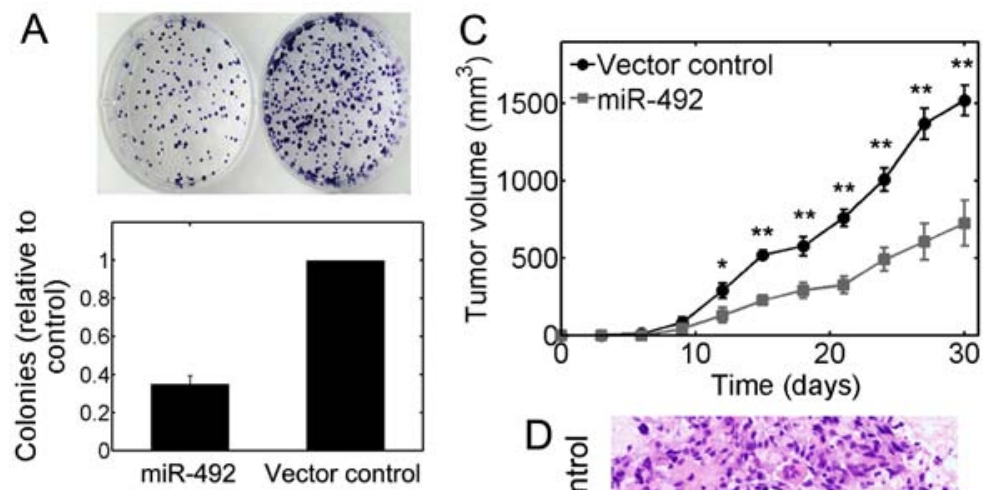

miR-492 Vector control
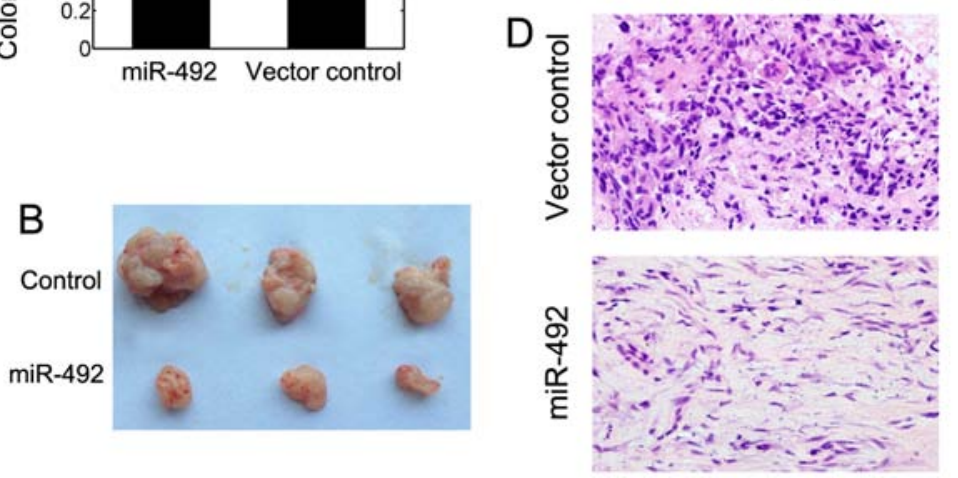

Figure 3. miR-492 inhibits tumorigenicity in vivo. (A) Colony formation of control or miR-492 expression vector U-2 osteosarcoma (OS) cells. Quantified results are shown below. (B) Representative xenografts of nude mice. (C) The growth of solid tumors in mice injected with U-2 OS cells transfected with either vector control or miR-492 vector. ${ }^{*} \mathrm{P}<0.05,{ }^{* *} \mathrm{P}<0.01$. (D) By the end of the implantation, solid tumors were resected and subjected to immunostaining on paraffinembedded sections. 
A
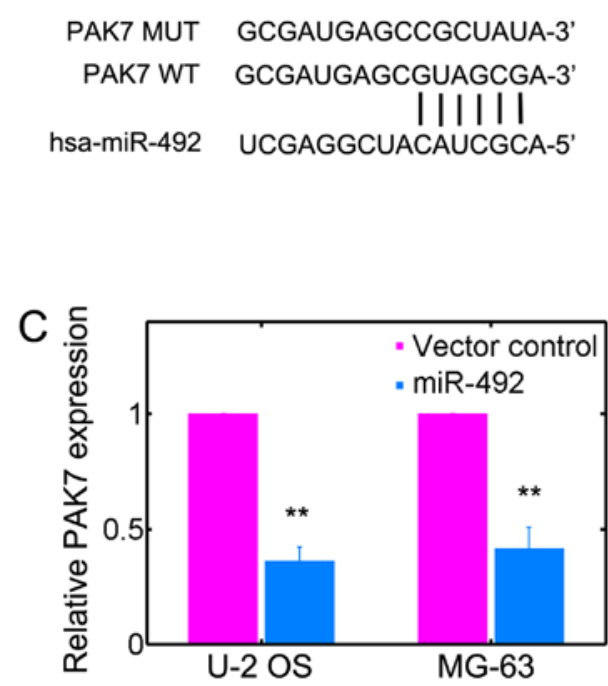

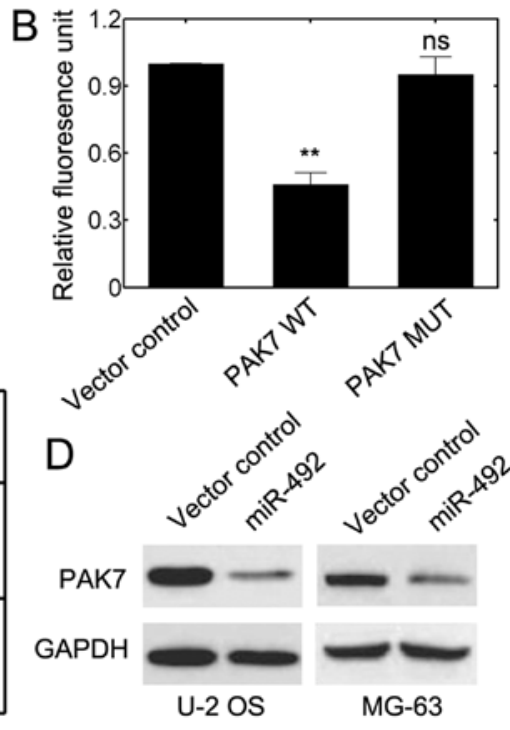

Figure 4. miR-492 targets PAK7 to inhibit tumorigenesis. (A) The 3'-untranslated region (3'-UTR) was predicted to be binding targets of miR-492. The mutant sequence is also shown above. (B) Luciferase activity of $293 \mathrm{~T}$ cells transfected with control vector, wild-type or mutant PAK7 plasmids. (C) Relative expression of PAK7 in U-2 OS and MG-63 cells transfected with either control vector or miR-492 expression vectors. ${ }^{* *} \mathrm{P}<0.01$. (D) Western blot analysis to determine the protein level of PAK7 in U-2 OS and MG-63 cells. Results are representative of triplicate experiments. ${ }^{* *} \mathrm{P}<0.01$; ns, not significant.
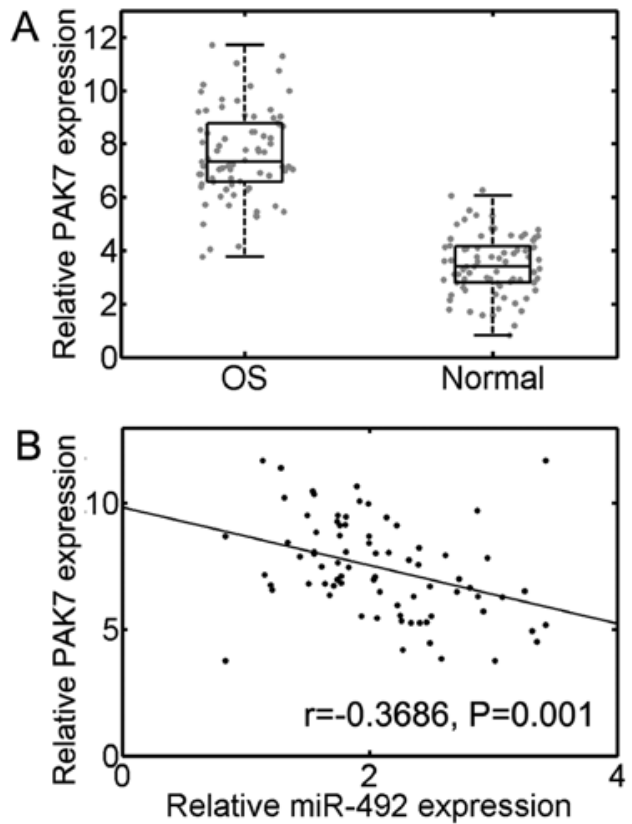

Figure 5. Correlation between miR-492 and PAK7 in osteosarcoma (OS) specimens. (A) expression of PAK7 in 77 OS samples and paired normal adjacent tissues quantified by RT-qPCR. (B) Pearson correlation between miR-492 expression and PAK7 transcripts in 77 OS samples. $\mathrm{r}=-0.3686, \mathrm{P}=0.001$.

miR-492 in modulating tumor formation in vivo, $10^{5} \mathrm{U}-2$ OS cells stably transfected with lentiviral miR-492 or lentiviral controls were subcutaneously injected into nude mice and the growth of solid tumors was evaluated every 3 days. The volume of solid tumors was significantly decreased in the groups injected with miR-492-transfected cells (Fig. 3B). A smaller tumor size was also evident in the groups injected with cells overexpressing miR-492 during a 30-day evaluation period (Fig. 3C). The Ki-67 immnostaining results further indicated that the growth of OS tumors was strongly inhibited by inducing miR-492 overexpression (Fig. 3D). Taken together, these results suggest a tumor suppressive role for miR-492 in OS in vivo.

$P A K 7$ is the target of $m i R-492$. To unravel the potential target for miR-492, we used online databases, such as microRNA.org (http://www.microrna.org/microrna/home.do), DIANA-microT (http://diana.imis.athenainnovation.gr/DianaTools/index.php?r= microT_CDS/index) and MIRDB (www.mirdb.org). It was suggested that $P A K 7$ may be the target of miR-492 (Fig. 4A). We further investigated whether miR-492 has a direct effect on $P A K 7$ using luciferase reporter assay. The complimentary sites on $P A K 7$ for miR-492 base pairing were mutated (Fig. 4A). We noted that miR-492 transfection substantially lowered the luciferase reporter activities in the PAK7 WT group (Fig. 4B). However, miR-492 failed to inhibit the luciferase activity in cells harboring PAK7 MUT (Fig. 4B). Furthermore, the transcript level of PAK7 was significantly downregulated by transfection iwth miR-492 precursor plasmids in U-2 OS and MG-63 cells (Fig. 4C). Western blot analysis further consolidated that the protein levels of PAK7 were consistently reduced with miR-492 transfection (Fig. 4D). These results support the prediction that miR-492 targets PAK7 in OS cells.

Level of miR-492 inversely correlates with PAK7 in OS. To further identify whether PAK7 is indeed downregulated in OS specimens, we carried out RT-qPCR analysis. The results suggested that $P A K 7 \mathrm{mRNA}$ expression was significantly upregulated in OS tissues compared to normal adjacent tissues ( $\mathrm{n}=77, \mathrm{P}<0.001$; Fig. 5A). Moreover, we further identified a significant inverse correlation between miR-492 and PAK7 transcript levels in OS samples $(\mathrm{r}=-0.3686, \mathrm{P}=0.001$; Fig. 5B). Taken together, our results suggest a tumorigenic role for PAK7 and establish an inverse correlation between miR-492 and PAK7. 


\section{Discussion}

The occurrence of tumors is a complex process and is associated with multiple factors. The dysregulation of miRs may also render tumor cells with proliferative advantage, resulting in uncontrolled growth, as well as other malignant characteristics (31). The exact role of miRs in tumorigenesis is undetermined and is possibly ascribed to the tumor microenvironment (32). Another reason may be due to the fact that miRs are usually located in the fragile locus of genes (33). Studies have focused on the role of $\mathrm{miR}$ in order to establish significant linkage between miRs and carcinogenesis $(34,35)$. Therefore, the identification of an association between miRs and tumorigenic factors may not only provide fruitful insight into diagnosis, but may also shed light on more effective therapeutic interventions.

In present study, we found that miR-492 can function as a tumor suppressor miR in OS. OS specimens usually showed reduced miR-492 expression compared with normal osteoblast cell lines. The ectopic expression of miR-492 using a lentiviral system effectively inhibited tumor growth, and the migration and invasion of OS cell lines. Further identification of miR-492 targets confirmed that PAK7 may be the candidate which was then experimentally verified. Moreover, PAK7 expression in OS also showed a significantly inverse correlation with miR-492, therefore further clarifying a tumor suppressor role for miR-492. The function of miR-492 has been reported in some cancer types. For instance, Jiang et al found that miR-492 promoted tumorigenesis in HCC by targeting PTEN (36). The miR-492-mediated effect was reversed by the addition of AKT inhibitor in HCC cell lines (36). Another study showed that miR-492 suppressed SOX7 expression and regulated the proliferation of breast cancer cell lines (17). However, a lower miR-492 expression contributes to oxaliplatin (a chemotherapeutic drug) resistance by elevating CD147 expression in LS174T/L-OHP colon cancer cell lines, implying that miR-492 could function similar to tumor suppressor (37). Therefore, whether miR-492 plays a tumor suppressor role or acts as an oncogenic factor is still elusive. Additionally, to date, there is no study focusing on the role of miR-492 in OS. In the present study, we argued that miR-492 can serve as a tumor suppressive factor as least in OS cells. As a result, our experiments clarified a novel role of miR-492 in OS and provide further knowledge about the functions of miR-492 in tumorigenesis.

The serine/threonine protein kinase, PAK7, was later identified as a putative miR-492 target suggesting that miR-492 can exert its function by suppressing PAK7. PAK7 has recently been predicted to be a potential biomarker in non-small cell lung cancer and OS $(11,38)$. Moreover, the aberrant expression of PAK7 is also evident in gastric cancer, suggesting that PAK7 may be critically important in promoting tumor progression (9). It has been previously demonstrated that PAK7 can support cell mobility and proliferation by activating survival pathways (11). The apoptotic signaling is also inhibited due to the mitochondria localization of PAK7 (40). In OS cells, the knockdown of PAK7 can also favor the induction of apoptosis. It can de-sensitize the apoptotic potential induced by camptothecin and $\mathrm{C} 2$-ceramide by phosphorylating $\mathrm{BAD}$, a pro-apoptotic member of the BCL-2 family at Ser-112 (11). PAK7 has also been shown to mediate cisplatin resistance and contributes to the progression of esophageal cancer (40). The mechanism was largely ascribed to the E2F1-induced transcriptional upregulation of PAK7 via Aurora-A activation (40). PAK7 has also been shown to serve as a biomarker for the proliferation of pancreatic cancer cell xenografts (12). Taken together, these findings strongly suggest an oncogenic role for PAK7 in various types of cancers. Therefore, targeting PAK7 for post-transcriptional regulation by miR-492 may be an effective strategy to efficiently attenuate the development and progression of OS.

In conclusion, in this study, we identified a tumor suppressive function of miR-492, at least in OS, by targeting PAK7. Since PAK7 has been shown to be extensively upregulated in various types of tumor and substantially promotes tumor progression, silencing PAK7 expression by miR-492 may prove to be an effective strategy with which to suppress the malignancy of OS. The intricate correlation between miR-492 and PAK7 needs to be investigated in more detail in order to further unravel the hidden layer of complexity in carcinogenesis and provide insight into diagnosis and pharmaceutical intervention.

\section{References}

1. Friedrich P, Ortiz R, Strait K, Fuentes S, Gamboa Y, Arambú I, Ah-Chu-Sanchez M, London W, Rodríguez-Galindo C, Antillón-Klussmann F, et al; Central American Association of Pediatric Hematologists Oncologists AHOPCA: Pediatric sarcoma in Central America: outcomes, challenges, and plans for improvement. Cancer 119: 871-879, 2013.

2. Mirabello L, Troisi RJ and Savage SA: International osteosarcoma incidence patterns in children and adolescents, middle ages and elderly persons. Int J Cancer 125: 229-234, 2009.

3. Morrow JJ and Khanna C: Osteosarcoma genetics and epigenetics: emerging biology and candidate therapies. Crit Rev Oncog 20: 173-197, 2015.

4. Admassi D: Osteosarcoma of medial cuniform bone. Ethiop Med J 47: 305-308, 2009.

5. Husmann K, Ducommun P, Sabile AA, Pedersen EM, Born W and Fuchs B: Signal transduction and downregulation of C-MET in HGF stimulated low and highly metastatic human osteosarcoma cells. Biochem Biophys Res Commun 464: 1222-1227, 2015.

6. Lee JA, Kim MS, Kim DH, Lim JS, Park KD, Cho WH, Song WS, Lee SY and Jeon DG: Postoperative infection and survival in osteosarcoma patients. Ann Surg Oncol 16: 147-151, 2009.

7. Martin H, Mali RS, Ma P, Chatterjee A, Ramdas B, Sims E, Munugalavadla V, Ghosh J, Mattingly RR, Visconte V, et al: Pak and Rac GTPases promote oncogenic KIT-induced neoplasms. J Clin Invest 123: 4449-4463, 2013.

8. Melzer J, Kraft KF, Urbach R and Raabe T: The p21-activated kinase $\mathrm{Mbt}$ is a component of the apical protein complex in central brain neuroblasts and controls cell proliferation. Development 140: 1871-1881, 2013.

9. Gu J, Li K, Li M, Wu X, Zhang L, Ding Q, Wu W, Yang J, Mu J, Wen $\mathrm{H}$, et al: A role for $\mathrm{p} 21$-activated kinase 7 in the development of gastric cancer. FEBS J 280: 46-55, 2013.

10. Zhang HH, Zhang ZY, Che CL, Mei YF and Shi YZ: Array analysis for potential biomarker of gemcitabine identification in non-small cell lung cancer cell lines. Int J Clin Exp Pathol 6: 1734-1746, 2013.

11. Han K, Zhou Y, Gan ZH, Qi WX, Zhang JJ, Fen T, Meng W, Jiang L, Shen Z and Min DL: p21-activated kinase 7 is an oncogene in human osteosarcoma. Cell Biol Int 38: 1394-1402, 2014.

12. Giroux V, Iovanna JL, Garcia S and Dagorn JC: Combined inhibition of PAK7, MAP3K7 and CK2alpha kinases inhibits the growth of MiaPaCa2 pancreatic cancer cell xenografts. Cancer Gene Ther 16: 731-740, 2009.

13. Gong W, An Z, Wang Y, Pan X, Fang W, Jiang B and Zhang H: P21-activated kinase 5 is overexpressed during colorectal cancer progression and regulates colorectal carcinoma cell adhesion and migration. Int J Cancer 125: 548-555, 2009.

14. Fromm B, Billipp T, Peck LE, Johansen M, Tarver JE, King BL, Newcomb JM, Sempere LF, Flatmark K, Hovig E, et al: A uniform system for the annotation of vertebrate microRNA genes and the evolution of the human microRNAome. Annu Rev Genet 49: 213-242, 2015. 
15. Bartel DP: MicroRNAs: target recognition and regulatory functions. Cell 136: 215-233, 2009.

16. Bai Y,Li J,Li J, Liu Y and Zhang B: miR-615 inhibited cell proliferation and cell cycle of human breast cancer cells by suppressing of AKT2 expression. Int J Clin Exp Med 8: 3801-3808, 2015.

17. Shen F, Cai WS, Feng Z, Li JL, Chen JW, Cao J and Xu B: miR-492 contributes to cell proliferation and cell cycle of human breast cancer cells by suppressing SOX7 expression. Tumour Biol 36: 1913-1921, 2015.

18. Cai K, Shen F, Cui JH, Yu Y and Pan HQ: Expression of miR-221 in colon cancer correlates with prognosis. Int J Clin Exp Med 8: 2794-2798, 2015.

19. Cheng CJ, Bahal R, Babar IA, Pincus Z, Barrera F, Liu C, Svoronos A, Braddock DT, Glazer PM, Engelman DM, et al MicroRNA silencing for cancer therapy targeted to the tumour microenvironment. Nature 518: 107-110, 2015.

20. Yanaihara N, Caplen N, Bowman E, Seike M, Kumamoto K Yi M, Stephens RM, Okamoto A, Yokota J, Tanaka T, et al: Unique microRNA molecular profiles in lung cancer diagnosis and prognosis. Cancer Cell 9: 189-198, 2006.

21. Jiang J, Gusev Y, Aderca I, Mettler TA, Nagorney DM, Brackett DJ, Roberts LR and Schmittgen TD: Association of MicroRNA expression in hepatocellular carcinomas with hepatitis infection, cirrhosis, and patient survival. Clin Cancer Res 14: 419-427, 2008.

22. Chen YJ, Wu H, Zhu JM, Li XD, Luo SW, Dong L, Liu TT and Shen XZ: MicroRNA-18a modulates P53 expression by targeting IRF2 in gastric cancer patients. J Gastroenterol Hepatol 31: 155-163, 2016.

23. Peng J: miR-638, a novel tumor suppressor for triple-negative breast cancer. ProQuest/UMI, 2014.

24. Su J, Wang Q, Liu Y and Zhong M: miR-217 inhibits invasion of hepatocellular carcinoma cells through direct suppression of E2F3. Mol Cell Biochem 392: 289-296, 2014.

25. He C, Xiong J, Xu X, Lu W, Liu L, Xiao D and Wang D: Functional elucidation of miR-34 in osteosarcoma cells and primary tumor samples. Biochem Biophys Res Commun 388: 35-40, 2009.

26. Li T, Lu YY, Zhao XD, Guo HQ, Liu CH, Li H, Zhou L, Han YN, Wu KC, Nie YZ, et al: MicroRNA-296-5p increases proliferation in gastric cancer through repression of Caudal-related homeobox 1. Oncogene 33: 783-793, 2014.

27. Liu X, Zhang J, Xie B, Li H, Shen J and Chen J: MicroRNA-200 family profile: a promising ancillary tool for accurate cancer diagnosis. Am J Ther 23: e388-e397, 2016.
28. Lewis BP, Shih IH, Jones-Rhoades MW, Bartel DP and Burge CB: Prediction of mammalian microRNA targets. Cell 115: 787-798, 2003.

29. Wong $\mathrm{N}$ and Wang $\mathrm{X}$ : miRDB: an online resource for microRNA target prediction and functional annotations. Nucleic Acids Res 43: D146-D152, 2015.

30. Maragkakis M, Reczko M, Simossis VA, Alexiou P, Papadopoulos GL, Dalamagas T, Giannopoulos G, Goumas G, Koukis E, Kourtis K, et al: DIANA-microT web server: elucidating microRNA functions through target prediction. Nucleic Acids Res 37: W273-276, 2009.

31. Leonardo TR, Schultheisz HL, Loring JF and Laurent LC: The functions of microRNAs in pluripotency and reprogramming. Nat Cell Biol 14: 1114-1121, 2012.

32. Hayes J, Peruzzi PP and Lawler S: MicroRNAs in cancer: biomarkers, functions and therapy. Trends Mol Med 20: 460-469, 2014.

33. Di Leva G, Garofalo M and Croce CM: MicroRNAs in cancer. Annu Rev Pathol 9: 287-314, 2014

34. Roderburg $\mathrm{C}$ and Luedde T: Circulating microRNAs as markers of liver inflammation, fibrosis and cancer. J Hepatol 61: 1434-1437, 2014.

35. Yang Y, Xing Y, Liang C, Hu L, Xu F and Chen Y: Crucial microRNAs and genes of human primary breast cancer explored by microRNA-mRNA integrated analysis. Tumour Biol 36: 5571-5579, 2015.

36. Jiang J, Zhang Y, Yu C, Li Z, Pan Y and Sun C: MicroRNA-492 expression promotes the progression of hepatic cancer by targeting PTEN. Cancer Cell Int 14: 95, 2014.

37. Peng L, Zhu H, Wang J, Sui H, Zhang H, Jin C, Li L, Xu T and Miao R: miR-492 is functionally involved in oxaliplatin resistance in colon cancer cells LS174T via its regulating the expression of CD147. Mol Cell Biochem 405: 73-79, 2015.

38. Markou A, Sourvinou I, Vorkas PA, Yousef GM and Lianidou E: Clinical evaluation of microRNA expression profiling in non small cell lung cancer. Lung Cancer 81: 388-396, 2013.

39. Wells CM and Jones GE: The emerging importance of group II PAKs. Biochem J 425: 465-473, 2010.

40. He S, Feng M, Liu M, Yang S, Yan S, Zhang W, Wang Z, Hu C, Xu Q, Chen L, et al: P21-activated kinase 7 mediates cisplatin-resistance of esophageal squamous carcinoma cells with Aurora-A overexpression. PLoS One 9: e113989, 2014. 